Pacific Journal of Mathematic 


\title{
ON MATRICES WITH A RESTRICTED NUMBER OF DIAGONAL VALUES
}

\author{
J. E. H. ElliotT
}

This note confirms the following conjecture of Marcus: Let $A=\left(a_{i j}\right)$ be an $n \times n$ matrix of strictly positive entries with at most $(n-1)$ distinct diagonal values, then $A$ is singular. We also show that there exist matrices with strictly positive entries with $n$ diagonal values which are nonsingular.

Definitions. If $A$ is an $n \times n$ matrix and $\sigma$ is a permutation of $\{1,2, \cdots, n\}$, then the product $a_{1, \sigma(1)} \cdot a_{2, \sigma(2)} \cdots a_{n, \sigma(n)}$ is called the $d i$ agonal of $A$ corresponding to $\sigma$.

If $A_{1}, A_{2}$ are two $n \times n$ matrices, then $A_{1}$ is called a diagonate of $A_{2}$ if $A_{1}$ can be obtained from $A_{2}$ by a finite number of operations of the following kinds:

(i) Multiplication of all entries of some row, (or column) by some $c>0$.

(ii) Interchange of any two rows (or columns).

The notation $A[\mu \mid \gamma], A(\mu \mid \gamma)$ is that of [1].

Preliminary Remarks. ( i ) The property of being a diagonate is an equivalence relation.

(ii) If a matrix is singular (nonsingular), then each of its diagonates is singular (nonsingular).

(iii) If a matrix $A_{1}$ has diagonal values $\rho_{1}<\rho_{2}<\cdots<\rho_{r}$ then a diagonate $A_{2}$ of $A_{1}$ has diagonal values $k \rho_{1}<k \rho_{2}<\cdots k \rho_{r}$, where $k=k\left(A_{2}\right)$, and $\left|\operatorname{det} A_{1}\right|=\left|k \operatorname{det} A_{2}\right|$.

(iv) If a matrix has strictly positive (positive) entries, then each of its diagonates has strictly positive (positive) entries.

LEMMA. If $X=\left(x^{e(i, j)}\right)$ is an $n \times n$ matrix with entries in an extension $F(x)$ of the real field $F$, where $e(i, j)$ are nonnegative rational integers $i, j=1,2, \cdots n$ and $e(1, j)=0$ for $j=1,2, \cdots n$, then

$\operatorname{det} X=(x-1)^{n-1} g(x)$, where $g(x)$ is a polynomial in $x$ with rational integral coefficients.

The proof of the lemma is by induction. The result is trivial for $n=2$. The result is therefore assumed to hold for all $n<N$, and $N>2$. If $n=N$, subtracting the first row of $X$ from the second and expanding $X$ by its second row, we have 


$$
\operatorname{det} X=\sum_{j=1}^{n}(-1)^{j}\left\{x^{e(2, j)}-1\right\} \operatorname{det} X(2 \mid j) ;
$$

but each of the matrices $X(2 \mid j)$ is of the form of the matrix of the hypothesis, and therefore by the induction assumption we have

$$
\operatorname{det} X(2 \mid j)=(x-1)^{n-2} g_{j}(x),
$$

where $g_{j}(x)$ is a polynomial in $x$ with rational integral coefficients. Thus

$$
\operatorname{det} X=\sum_{j=1}^{n}(-1)^{j}\left\{x^{e(2, j)}-1\right\}(x-1)^{n-2} g_{j}(x)=(x-1)^{n-1} g(x) .
$$

We are now in a position to prove the conjecture.

The conjecture is proved below by induction on the order of the matrix. Therefore we first prove the theorem for a $3 \times 3$ matrix.

THEOREM 1. If $A_{\alpha}$ is a $3 \times 3$ matrix of strictly positive entries with at most two distinct diagonal values, then $A_{\alpha}$ is singular.

To prove this, it is supposed that $A_{\alpha}$ is nonsingular: then there exist nonsingular minors $A_{\alpha}(i \mid j)$ with diagonal values $\rho_{1}(i, j)<\rho_{2}(i, j)$. Consequently there exists a diagonate $A_{\beta}$ of $A_{\alpha}$ where the ratio $\lambda=$ $\rho_{2}(1,1) / \rho_{1}(1,1)$ is maximal, and $A_{\beta}$ has two distinct diagonal values $\gamma_{11} \rho_{1}(1,1), \lambda \gamma_{11} \rho_{1}(1,1)$. Thus there exists a diagonate $A_{r}$ or $A_{\beta}$ such that $\gamma_{3 i}=\gamma_{i 3}=1$ for $i=1,2,3, \gamma_{22}=\lambda$ where $A_{\gamma}=\left(\gamma_{i j}\right)$. Since $A_{\alpha}$ is nonsingular $A_{r}$ is also nonsingular, and $\lambda$ retains its maximality property in $A_{\gamma}$. Now if $d$ is the entry $A_{\gamma}(i, 3 \mid j, 3)$ where $i \neq 3, j \neq 3$, then $\gamma_{i j} d$ and $d$ are both diagonal values, so consideration of their ratio shows that $\gamma_{i j}=\lambda, 1$ or $\lambda^{-1}$. Consideration of the minors $A_{\gamma}[1,3 \mid 2,3]$ and $A_{\gamma}[1,2 \mid 2,3]$ shows, by the maximality property of $\lambda$, that $\gamma_{21}, \gamma_{12}$ are no less than 1 . Putting $\gamma_{11}=1$ therefore, since no columns (rows) are equal, yields $\gamma_{21}=\gamma_{12}=\lambda$. This gives a contradiction, as the matrix now has three distinct diagonal values $1, \lambda$ and $\lambda^{2}$. If $\lambda_{11}=\lambda^{-1}$, then $A_{\gamma}$ has distinct diagonal values $\lambda, \lambda^{-1}$, and a consideration of their ratio leads to a contradiction. We must therefore have $\gamma_{11}=\lambda$, and so $A_{\gamma}$ has diagonal values $\lambda, \lambda^{2}$. However, since $\gamma_{21}$ and $\gamma_{12}$ are also diagonal values each equal to 1 , or $\lambda$, then $\gamma_{12}=\gamma_{21}=\lambda$, and again since $A_{r}$ is nonsingular we have a contradiction. But this has exhausted all possibilities for the value of $\gamma_{11}$ and so the proof of Theorem 1 is complete.

We are now in a position to prove the conjecture for all $n$. 
THEOREM 2. If $A_{\alpha}$ is an $n \times n$ matrix of strictly positive entries with at most $(n-1)$ distinct diagonal values then $A_{\alpha}$ is singular.

The proof of this theorem is by induction on $n$. The result is trivial for $n=2$, and it has been proved for $n=3$. Therefore we assume the theorem to hold for all $n<N$, where $N>3$. It is supposed that $A_{\alpha}$ is an $N \times N$ matrix of the diagonate class $A=\left\{A_{\omega} ; \omega \in \Omega\right\}$. The proof is by contradiction; we assume that $A_{\alpha}$ is nonsingular. By the Expansion Theorem of Laplace, [1], given two rows $r, s$ of $A_{\alpha}$ there exist two columns $t, u$ such that $A_{\alpha}[r, s \mid t, u)$ and $A_{\alpha}(r, s \mid t, u)$ are both nonsingular. It then follows from the induction assumption that the matrix $A_{\alpha}[r, s \mid t, u]$ has at least two distinct diagonal values $\mu_{1}<\mu_{2}$, and the matrix $A_{\alpha}(r, s \mid t, u)$ has at least $(N-2)$ distinct diagonal values $\rho_{1}<\rho_{2}<\cdots<\rho_{N-2}$. Therefore $A_{\alpha}$ must have at least the $(N-1)$ distinct diagonal values $\mu_{1} \rho_{1}<\mu_{2} \rho_{1}<\mu_{2} \rho_{2}<\cdots<\mu_{2} \rho_{N-2}$. However $A_{\alpha}$ has at most $N-1$ distinct diagonal values, and so these diagonal values must also be exactly the values

$$
\mu_{1} \rho_{1}<\mu_{1} \rho_{2}<\cdots<\mu_{1} \rho_{N-2}<\mu_{2} \rho_{N-2} .
$$

It therefore follows that

$$
\frac{\mu_{2}}{\mu_{1}}=\frac{\rho_{2}}{\rho_{1}}=\cdots=\frac{\rho_{N-2}}{\rho_{N-3}}>1 .
$$

Hence if $\lambda$ denotes the ratio $\mu_{2} / \mu_{1}$, then the matrix $A_{\alpha}$ has for its $(N-1)$ distinct diagonal values exactly the $(N-1)$ diagonal values $c<\lambda c<\cdots<\lambda^{N-2} c$, where $c=\mu_{1} \rho_{1}$. Now there exists $A_{\beta}=\left(a_{i j}\right) \in A$ such that $a_{i 1}=a_{1 i}=1$ for $i=1,2, \cdots, N$, and $A_{\beta}$ has diagonal values $k<\lambda k<\cdots<\lambda^{N-2} k$ for some $k>0$. If $d$ is any diagonal value of $A_{\beta}(1, i \mid 1, j)$ then $a_{i j} d$, and $d$ are diagonal values of $A_{\beta}$ and thus $a_{i j}$ is an integral power of $\lambda$. A division of the $j$-th row of $A_{\beta}$ by $\min \left\{a_{i j} ; i=1,2, \cdots N\right\}$ for $j=2,3, \cdots N$, yields a matrix $A_{r} \in A$, $A_{r}=\left(\gamma_{i j}\right)$ such that $\gamma_{i j}=\lambda^{e(i, j)}$ where $e(i, j)$ is a nonnegative rational integer for $i, j=1,2, \cdots N, e(1, j)=0$ for $j=1, \cdots N$, and $A_{r}$ has diagonal values

$$
\lambda^{h}<\lambda^{h+1}<\cdots<\lambda^{h+N-2} .
$$

Now let $E$ denote the $N \times N$ matrix with $(i, j)$ th entry $x^{e(i, j)}$, where $x$ is transcendental over the real field. By the lemma, det $E=$ $(x-1)^{N-1} g(x)$, where $g(x)$ is a polynomial with rational integral coefficients. However $E$ has exactly the diagonal values

$$
x^{h}<x^{h+1}<\cdots<x^{h+N-2}
$$

and thus $\operatorname{det} E=x^{h}\left\{b_{0}+b_{1} x+\cdots b_{N-2} x^{N-2}\right\}=(x-1)^{N-1} g(x)$ where $b_{i}$, 
$i=0,1, \cdots N-2$ is a rational integer. This however implies that $b_{0}=b_{1}=\cdots=b_{N-2}=0$, and thus

$$
\operatorname{det} A_{r}=\lambda^{h}\left\{b_{0}+b_{1} \lambda+\cdots+b_{N-2} \lambda^{N-2}\right\}=0 \text {. }
$$

We therefore have $A_{\alpha}, A_{\gamma}$ two matrices of the same diagonate class one nonsingular and one singular. This is the required contradiction which completes the proof of the conjecture. We can also conclude the result below.

CoROLlary. If an $n \times n$ matrix $A$ with strictly positive entries has at most $r$ distinct diagonal values and $r<n$, then $\operatorname{rank}(A) \leqq r$.

To show that an $n \times n$ matrix of strictly positive entries need not be singular if it takes on as few as $n$ diagonal values, we may consider the $n \times n$ matrix $C=\left(c_{i j}\right)$, where $c_{i i}=k$ for $\mathrm{i}=2,3, \cdots n$, and $c_{i j}=\lambda$ otherwise; and where $k, \lambda$ are positive integers such that $k>\lambda$. Then $\operatorname{det} C=\lambda(k-\lambda)^{n-1} \neq 0$.

\section{REFERENCES}

1. M. Marcus and H. Minc, A Survey of Matrix Theory and Matrix Inequalities, Allyn and Bacon Inc., Boston, 1964.

Received July 25, 1968.

UNiversity COLlege, CORK 


\section{PACIFIC JOURNAL OF MATHEMATICS}

\section{EDITORS}

H. SAmelson

Stanford University

Stanford, California 94305

RICHARd PieRCe

University of Washington

Seattle, Washington 98105

\author{
J. DugundJI \\ Department of Mathematics \\ University of Southern California \\ Los Angeles, California 90007 \\ RICHARD ARENS \\ University of California \\ Los Angeles, California 90024
}

ASSOCIATE EDITORS
E. F. BECKENBACH
B. H. NeumanN
F. WOLF
K. YosHIDA

\section{SUPPORTING INSTITUTIONS}

UNIVERSITY OF BRITISH COLUMBIA

CALIFORNIA INSTITUTE OF TECHNOLOGY

UNIVERSITY OF CALIFORNIA

MONTANA STATE UNIVERSITY

UNIVERSITY OF NEVADA

NEW MEXICO STATE UNIVERSITY

OREGON STATE UNIVERSITY

UNIVERSITY OF OREGON

OSAKA UNIVERSITY

UNIVERSITY OF SOUTHERN CALIFORNIA
STANFORD UNIVERSITY

UNIVERSITY OF TOKYO

UNIVERSITY OF UTAH

WASHINGTON STATE UNIVERSITY

UNIVERSITY OF WASHINGTON

AMERICAN MATHEMATICAL SOCIETY CHEVRON RESEARCH CORPORATION TRW SYSTEMS

NAVAL WEAPONS CENTER 


\section{Pacific Journal of Mathematics}

\section{Vol. 35, No. $1 \quad$ September, 1970}

B. D. Arendt and C. J. Stuth, On the structure of commutative periodic semigroups ..................................... 1

B. D. Arendt and C. J. Stuth, On partial homomorphisms of semigroups .... 7

Leonard Asimow, Extensions of continuous affine functions ............ 11

Claude Elias Billigheimer, Regular boundary problems for a five-term recurrence relation ................................... 23

Edwin Ogilvie Buchman and F. A. Valentine, A characterization of the

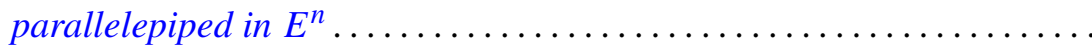
53

Victor P. Camillo, A note on commutative injective rings ............ 59

Larry Jean Cummings, Decomposable symmetric tensors ............. 65

J. E. H. Elliott, On matrices with a restricted number of diagonal values ... 79

Garth Ian Gaudry, Bad behavior and inclusion results for multipliers of type

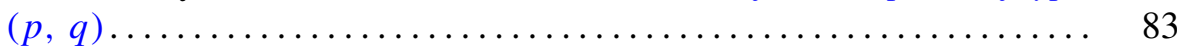

Frances F. Gulick, Derivations and actions .................. 95

Langdon Frank Harris, On subgroups of prime power index . . . . . . . . 117

Jutta Hausen, The hypo residuum of the automorphism group of an abelian

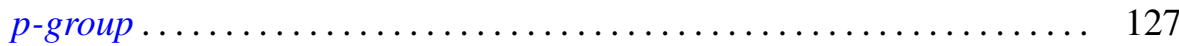

R. Hrycay, Noncontinuous multifuctions . ................... 141

A. Jeanne LaDuke, On a certain generalization of $p$ spaces ............ 155

Marion-Josephine Lim, Rank preservers of skew-symmetric matrices...... 169

John Hathway Lindsey, II, On a six dimensional projective representation of the Hall-Janko group ................................... 175

Roger McCann, Transversally perturbed planar dynamical systems . . . . . 187

Theodore Windle Palmer, Real $C^{*}$-algebras ................... 195

Don David Porter, Symplectic bordism, Stiefel-Whitney numbers, and a

Novikov resolution ....................................... 205

Tilak Raj Prabhakar, On a set of polynomials suggested by Laguerre

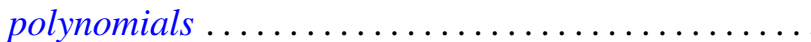

B. L. S. Prakasa Rao, Infinitely divisible characteristic functionals on locally convex topological vector spaces...................

John Robert Reay, Caratheodory theorems in convex product structures ...

Allan M. Sinclair, Eigenvalues in the boundary of the numerical range. .

David R. Stone, Torsion-free and divisible modules over matrix rings ....

William Jennings Wickless, A characterization of the nil radical of a

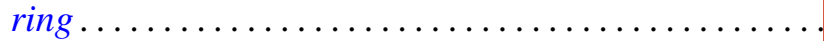

